\title{
Accuracy Versus Falsification Costs: The Optimal Amount of Evidence under Different Procedures
}

\author{
Winand Emons* \\ Universität Bern and CEPR \\ Claude Fluet $^{\star *}$ \\ Université du Québec à Montréal and CIRPEE
}

\begin{abstract}
An arbiter can decide a case on the basis of his priors or he can ask for further evidence from the two parties to the conflict. The parties may misrepresent evidence in their favor at a cost. The arbiter is concerned about accuracy and low procedural costs. When both parties testify, each of them distorts the evidence less than when they testify alone. When the fixed cost of testifying is low, the arbiter hears both, for intermediate values one, and for high values no party at all. The arbiter's ability to remain uninformed as well as sequential testifying makes it more likely that the arbiter requires evidence. (JEL D82, K41, K42)
\end{abstract}

\section{Introduction}

How much testimony should an arbiter require when he knows that the parties to the conflict spend considerable resources to misrepresent evidence in their favor? When he hears no witnesses, no resources are wasted on fabricating evidence, yet the judge's adjudication will be erroneous, leading to a social loss from inaccurate decisions. If parties testify, the decision will be more accurate, yet, resources are wasted on fabricating evidence. Requiring, for example, testimony from two rather than one party will lead to a duplication of the costs to produce misleading information. The purpose of this article is to analyze this trade-off between procedural costs and the benefits of truth finding.

An arbiter has to decide on an issue which we take to be a real number; for example, the adjudicated value are the damages that one party owes to the other. The defendant wants the damages to be small, whereas the plaintiff wants them to be large. The parties thus have conflicting interests. The arbiter

\footnotetext{
*Departement Volkswirtschaftslehre, Universität Bern, Bern, Switzerland. Email: winand. emons@vwi.unibe.ch.

**Université du Québec à Montréal and CIRPÉE, Montréal, Canada. Email: fluet.claude-denys (a)uqam.ca.

We thank Howard Chang, Bruno Deffains, Oliver Fabel, Marie-Cécile Fagart, Christian Ghiglino, Jeong-Yoo Kim, Tracy Lewis, Nicolas Marceau, Gerd Mühlheusser, Harris Schlesinger, Dezsö Szalay, and two referees for helpful comments. The usual disclaimer applies.
}

The Journal of Law, Economics, \& Organization, Vol. 25, No. 1, doi: $10.1093 /$ jleo/ewm046 
can decide the case solely on the basis of his priors. Alternatively, he can ask for further evidence from the parties to the conflict.

Both parties know the actual realization of the damages. Presenting evidence involves a fixed cost. Moreover, the parties can boost the evidence in either direction. Distorting the evidence is, however, costly. The further a party moves away from the truth, the higher the cost; for example, expert witnesses charge more, the more they distort the truth.

The arbiter first announces whether he wants to hear no, one, or both parties. Given he hears testimony, the arbiter updates his beliefs about the true value. Then he adjudicates. The arbiter chooses the amount of testimony and the adjudicated value so as to minimize the sum from the loss of inaccurate adjudication plus the parties' submission costs. He thus trades-off the social benefits of truth finding against the cost of obtaining evidence.

We first look at the case where, if both parties testify, they do so simultaneously. When the arbiter decides to hear no party, he adjudicates the prior mean. When he decides to hear one party, her testimony is monotonic in the true value; yet, depending on who testifies, she overstates respectively understates the true value. Accordingly, the equilibrium is revealing but it involves falsification. The arbiter rationally corrects for the exaggerated amount and adjudicates the true value. Stated differently because the marginal cost of slightly distorting the truth is negligible but the marginal return is not, the arbiter expects some falsification, leading parties to do so systematically.

When both parties submit, both testimonies are monotonic and involve falsification: one party overreports whereas the other underreports. The arbiter corrects for this by taking an average of the exaggerated testimonies. When the arbiter hears only one party, this party lies more than her extent of lying when both parties submit. When only one party presents evidence, the arbiter gives more weight to the party's submission, thereby inducing her to falsify more. As a result, confronting the parties in adversarial hearings induces either of them to distort the evidence less than when only one party is heard. Yet, when both parties are heard, both are involved in boosting the evidence.

The optimal number of parties to submit evidence depends on the value of information and the cost of obtaining evidence. If the value of information is above some threshold, the arbiter hears both parties given the fixed cost of presenting evidence is small. For intermediate values of the fixed costs he goes for one party and for large values he hears no party at all. Even when the cost of obtaining evidence is accounted for it may, therefore, still be optimal to hear both parties: the duplication of the fixed submission costs is more than compensated by the lower cost of boosted evidence.

Next we consider whether alternative institutional arrangements can improve the trade-off between accuracy in adjudication and falsification. We first study the role of commitment by the arbiter. We look at the case where the arbiter can commit to a probability of not reading the reports he has asked for. When he remains uninformed, he adjudicates the prior mean. Compared to the noncommitment case, the parties now falsify less: boosting one's claim has less influence on the arbiter's decision because the reports may not be read. 
When they are read, the arbiter infers the truth and adjudicates accordingly. Yet, adjudication is not always accurate because reports are sometimes ignored. Compared to the noncommitment case, it is now less likely that the arbiter will go for no testimony.

In the second alternative arrangement, we revert to noncommitment but allow parties to submit sequentially such that the second party can react to the first party's submission. Here we construct an equilibrium where both parties report truthfully. Exaggerated claims by the first party provoke the second party to boost her own claims. Given this threat of retaliation, the first party does not falsify in the first place. Compared to the other two scenarios, joint submissions are now more attractive.

We thus develop a simple framework which allows us to determine for different institutional arrangements when an arbiter should hear two, one, or no party at all. The lower the fixed costs of making a presentation or the more inaccurate the arbiter's prior information, the more parties should be heard in the proceedings. The ability to commit to remain uninformed leads to less falsification and makes hearing testimony more attractive. With sequential hearings, it is possible to have nonfalsified testimonies, which makes joint submissions even more attractive.

It is standard in the literature to view accuracy in adjudication and procedural economy as the objectives at which legal procedures should aim. Adversarial systems of discovery clearly motivate parties to provide evidence. Nevertheless, they are often criticized (e.g., Tullock 1975, 1980) for yielding excessive expenditures through unnecessary duplication and costly overproduction of misleading information.

We refer to legal procedures for concreteness. However, the same issues arise in regulatory or administrative hearings as well as in many other contexts. For example, Milgrom (1988) argues that those best informed of the consequences of alternative decisions are also often the ones most affected by them. Therefore, organizations face a trade-off between eliciting useful information and limiting the wasteful "influence activities," geared at purely redistributive aims, of those who inform decision makers.

Our contribution is to tackle the cost/accuracy trade-off as a signaling game on the basis of the so-called "costly state falsification" approach with costly testimonies as signals. ${ }^{1}$ The decision maker's problem is whether he should require both parties to signal, or only one, or none. Hearing only one party results in a one-sender signaling game with a continuum of types where a type is given by the true state of the world. Hearing both parties yields a multisender game with perfectly correlated types. Since signals are nonstochastic, the true sate is inferred in both procedures. Thus, we can focus on how much wasteful

1. The costly state falsification approach has been used exclusively in a principal-agent context. See Lacker and Weinberg (1989), Maggi and Rodríguez-Clare (1995), and Crocker and Morgan (1998). For example, the latter article analyzes the falsification of insurance claims. The agent is privately informed about the true value of the loss and is able to misrepresent this quantity at a cost. 
signaling each procedure entails. In addition, when both parties are heard, different arrangements are feasible, for example, sequential versus simultaneous submissions, resulting in different signaling expenditures.

One approach to court decision making views the trial outcome as an exogenous function of the litigants' levels of effort or expenditure by using socalled contest functions. See Cooter and Rubinfeld (1989) for a review of the earlier literature; later examples include Katz (1988), Farmer and Pecorino (1999), Bernardo et al. (2000), and Parisi (2002). In these articles adjudication is a zero-one variable where a party either wins or loses. Parties engage in a rent-seeking game, leading to excessive expenditures. Our approach differs in that the arbiter's adjudication rule is not specified exogenously: decisions are part of a perfect Bayesian equilibrium. In our setup, the arbiter is a sophisticated decision maker who understands the parties' incentives to "boost" the submitted evidence.

Our approach also differs from other expenditure-based models which consider guilty or innocent defendants; see, for example, Rubinfeld and Sappington (1987) and Sanchirico (2001). In the latter, a defendant's type is private information. The defendant's level of effort determines the probability that she will be found innocent, given the standard of proof. This probability function is exogenously given and differs between types. The arbiter minimizes the sum of the losses from type 1 and type 2 errors plus the defendant's expected effort cost with respect to the standard of proof and the penalty for conviction. When effort is not observable, both types of defendant provide effort, the innocent defendant more than the guilty one. The major difference to our setup is that the court faces just one defendant who can be of two types. Moreover, the court perfectly commits to a mechanism so that decisions are not ex post optimal given the court's updated beliefs. Rubinfeld and Sappington (1987) do not address the question of how many witnesses should be heard.

We also differ from another well-known strand of literature in which parties cannot falsify the verifiable evidence as such but are able to misrepresent it by disclosing only what they see fit; see Sobel (1985), Milgrom and Roberts (1986), Lewis and Poitevin (1997), and Shin (1998). Finally, our article is related to the literature comparing adversarial with inquisitorial procedures of truth finding; see Shin (1998), Dewatripont and Tirole (1999), and Palumbo (2001). In the inquisitorial system, a neutral investigator searches for evidence, and in the adversarial system, the parties to the conflict present the evidence. The last two articles compare the two procedures in terms of the costs to motivate agents to gather and produce verifiable information. By contrast, we look at the question how much testimony from interested parties should be used. Our judge or arbiter is therefore an active agent since he directs how the procedure will evolve. ${ }^{2}$

2. It is of course possible to interpret our cases where the judge hears no or one agent as inquisitorial and the case where he hears both parties as adversarial. Nevertheless, note that our judge has full control over whom he wants to hear, a feature typically associated with inquisitorial systems; see Posner (1973, 1999). 
Yet another approach can be found in Froeb and Kobayashi $(1996,2001)$ and Daughety and Reinganum (2000a, 2000b) who model the adversarial provision of evidence as a game in which two parties engage in strategic sequential search. In the first set of articles, the arbiter is assumed to be a naive decision maker who takes the average of the evidence submitted. In the second, court decisions are also non-Bayesian but with an adjudication rule satisfying reasonable symmetry assumptions. These modeling assumptions do not, therefore, allow a comparison of different procedures.

The article is organized as follows. In the next section we describe our basic setup. The following section derives the optimal procedures for the simultaneous submission case. In the subsequent section, we look at the alternative institutional arrangements. Section 5 concludes. Proofs are relegated to the Appendix.

\section{The Model}

The issue to be settled is the value of $x \in \mathbb{R}$. The adjudicator-regulatory commission, court, etc. - has prior beliefs represented by the density $f(x)$ with full support over the real line, mean $\bar{x}$, and variance $\sigma^{2}$. The arbiter's initial beliefs may be taken as being shaped from information publicly available at the beginning of the proceedings. ${ }^{3}$

The arbiter can adjudicate solely on the basis of his priors. Alternatively, he can require further evidence to be submitted from perfectly informed but selfinterested actors denoted $A$ and $B$. Party $A$ would like the adjudicated value of $x$ to be large, whereas party $B$ would like it to be small. For example, the adjudicated value may be the damages that should be paid to the plaintiff $A$ by the defendant $B$; in a divorce case, it may be the amount of support $A$ should get from $B$; in regulatory hearings about the rental charge for a local loop the incumbent wants the charge to be high whereas the entrant wants it to be low.

Submissions by the parties are costly. A submission is of the form "the value of the quantity at issue is $x_{i}, " i=A, B$. It should be thought of as a story or argument rendering $x_{i}$ plausible, together with the supporting documents, witnesses, etc. The cost of a presentation is

$c_{i}\left(x_{i}, x\right)=\gamma+0.5\left(x_{i}-x\right)^{2}, \quad i=A, B$,

where $\gamma \geq 0$. The actual value is $x$, which is observed by the party, and $x_{i}$ is the testimony or the statement submitted.

A distorting presentation is more costly than simply reporting the naked truth as it involves more fabrication. We take a quadratic function to capture the idea that the cost of misrepresenting the evidence increases at an increasing rate the further one moves away from the truth: it becomes more difficult to

3. We assume full support over the real line in order to avoid boundary conditions. The probability of extreme values of $x$ can be made, however, arbitrarily small. 
produce the corresponding documents or experts charge more the more they distort the truth. ${ }^{4}$

The parties' capacity to falsify - their "credibility" - is common knowledge. Total submission cost is $C=0$ if no evidence is required from the parties. It is $C=c_{i}$ if only party $i, i=A, B$, submits. Otherwise, it is $C=c_{A}+c_{B}$.

The arbiter is concerned about the loss from inaccuracy in adjudication and the parties' submission costs. Accordingly, there is a potential trade-off between procedural costs and the social benefits of correct adjudication. From the arbiter's perspective, the total social loss is

$L=l+C$

where $l$ is the loss from inaccurate adjudication or "error costs" and $C$ is total submission costs.

Let $\hat{x}$ denote the arbiter's decision. The loss from inaccurate adjudication is

$l(\hat{x}, x)=\theta(\hat{x}-x)^{2}$

where $\theta>0$ is the rate at which the arbiter trades off accuracy against submission costs. If the true value is adjudicated, error costs are zero. The more the decision errs in either direction, the higher the losses from inaccurate adjudication and such losses increase at an increasing rate the further one moves away from the truth. The loss $l$ should be interpreted as the societal cost of incorrect decisions. For instance in tort cases, incorrect adjudication may have an adverse effect on deterrence.

The setup is as follows. The arbiter announces whether he wants to hear no, one, or both parties. We denote this decision by $d \in\{N, S, J\}$, where $N$ stands for no party being heard, $S$ for only a single party being heard (this would specify which one), and $J$ for joint submissions. We will deal with no, single, and joint submissions in different subsections so that we omit an index for $d$ wherever possible.

Then the parties observe $x$. If asked to testify, the parties choose $x_{A}=x_{A}(x)$ and/or $x_{B}=x_{B}(x)$ so as to maximize $\pi_{A}$ and $\pi_{B}$ where

$$
\begin{aligned}
& \pi_{A}\left(\hat{x}, x_{A}, x\right)=\hat{x}-c_{A}\left(x_{A}, x\right) \text { and } \\
& \pi_{B}\left(\hat{x}, x_{B}, x\right)=-\hat{x}-c_{B}\left(x_{B}, x\right) .
\end{aligned}
$$

When a party is called upon to testify, she has to do so and incur the fixed cost. ${ }^{5}$ If the arbiter hears testimony, he updates his beliefs $\mu(x \mid \cdot)$ which denotes the

4. Using quadratic falsification costs is standard in the literature. Maggi and Rodríguez-Clare (1995) work with $c_{i}\left(x_{i}, x\right)=\gamma+\kappa\left(x_{i}-x\right)^{2}$ and interpret $\kappa$ as capturing the publicness of information. If $\kappa=0$, falsification is costless; therefore, information is purely private. As $\kappa$ increases, it becomes more costly to falsify information and for an arbitrarily large $\kappa$ the public information model obtains.

5. If a party can decline to testify, she may avoid the fixed cost. Declining to testify signals that the true value does not exceed some upper bound. The analysis of this possibility is the subject of work in progress. 
probability distribution over $x$ in the information set given by the testimony. He then adjudicates $\hat{x}:=\phi(\cdot)$ so as to minimize the expected loss $E_{\mu}(l \mid \cdot)$, where the expectation is taken under the beliefs $\mu$. The arbiter chooses $d$ so as to minimize the expected social loss $\bar{L}_{d}$. We focus on perfect Bayesian equilibria. We first solve each of the subgames given by $d \in\{N, S, J\}$ and then derive the equilibria of the entire game.

\section{Simultaneous Submissions}

In this section under joint submission, the parties choose their reports simultaneously. ${ }^{6}$

\subsection{No Party Submits}

Under procedure $d=N$, no party testifies and submission costs are therefore zero. The arbiter then minimizes expected error costs solely on the basis of the priors, that is, $\mu(\cdot)=f(\cdot)$, implying $\hat{x}=\bar{x}$. The expected total loss is $\bar{L}_{N}=\theta \sigma^{2}$. Obviously, $\theta \sigma^{2}$ is also the value of perfect information, given the accuracy $\sigma^{2}$ of the arbiter's prior information.

\subsection{One Party Submits}

Suppose the arbiter has asked party $A$ for her testimony and adjudicates $\hat{x}=\phi\left(x_{A}\right)$. Then $A$ maximizes her payoff $\pi_{A}$ and her strategy satisfies the first-order condition $x_{A}(x)=x+\phi^{\prime}\left(x_{A}\right)$. The arbiter's strategy satisfies $\phi\left(x_{A}\right)=E_{\mu}\left[x \mid x_{A}\right]$. This condition follows from the arbiter adjudicating so as to minimize error costs given his updated beliefs at the information set defined by the observation of $x_{A}$. We first show a preliminary result.

Lemma 1. Every equilibrium is revealing and in every equilibrium the testifying party falsifies.

If an equilibrium is not revealing, it must be the case that, say, party $A$ makes the same report $x_{A}$ for at least two different values of $x$. Since $A$ 's optimal testimony is the sum of the true $x$ plus the marginal effect of his report $\phi^{\prime}\left(x_{A}\right)$, this cannot be true. Given that the equilibrium is revealing, $\phi$ is strictly monotone in $x_{A}$ : different $x$ 's give rise to different $x_{A}$ 's to which the arbiter reacts to adjudicate the true value of $x$. Since $\phi^{\prime} \neq 0$ and the marginal cost of lying is zero around the true value, it follows that it pays for a party to falsify.

Since equilibria are revealing, error costs are zero. Yet the parties falsify, so that falsification costs are positive. We focus on minimum falsification cost equilibria. ${ }^{7}$ It turns out that they have a simple structure: if party $A$ is heard,

6. Under joint submissions, we have a multisender signaling game where both parties know the true state. Examples for such games can be found in Bagwell and Ramey (1991), Hetzendorf and Overgaard (2001), Fluet and Garella (2002), and Kim (2003).

7. Signaling games generally have multiple equilibria, as is well known, and it is common in the literature to select the least-cost separating equilibrium. See Riley (2001) for a survey. 
she always overstates the true value by 1 ; if, by contrast, party $B$ is heard, she always understates the true value by 1 . The judge's beliefs reflect the parties' testifying behavior and when adjudicating he subtracts or adds 1 to the reported value. In the proof, we characterize the entire set of revealing equilibria. Out of this set, we select the equilibrium where the extent of lying $\left|x_{i}-x\right|, i=A, B$ is minimal and is equals to 1 .

\section{Proposition 1.}

(i) If only party $A$ is heard, the unique minimum falsification cost equilibrium has strategies $x_{A}(x)=x+1, \phi\left(x_{A}\right)=x_{A}-1$, and beliefs

$\mu\left(x \mid x_{A}\right)= \begin{cases}1, & \text { for } x=x_{A}-1 ; \\ 0, & \text { otherwise. }\end{cases}$

The expected loss $\bar{L}_{S}=\gamma+0.5$.

(ii) If only party $B$ is heard, the unique minimum falsification cost equilibrium has strategies $x_{B}(x)=x-1, \phi\left(x_{B}\right)=x_{B}+1$, and beliefs

$\mu\left(x \mid x_{B}\right)= \begin{cases}1, & \text { for } x=x_{B}+1 \\ 0, & \text { otherwise }\end{cases}$

The expected loss $\bar{L}_{S}=\gamma+0.5$.

Note that this equilibrium involves as much falsification as would occur if the testifying party thought she was facing a "naive" arbiter, that is, one who believes the true $x$ to be equal to the party's submission. The proposition therefore shows, somewhat surprisingly, that there cannot be less falsification with a "smart" arbiter who is known to draw the correct inferences.

\subsection{Both Parties Submit}

Suppose the arbiter has asked both parties for their testimony and adjudicates $\hat{x}=\phi\left(x_{A}, x_{B}\right)$. Then $A$ maximizes her payoff $\pi_{\mathrm{A}}, B$ her payoff $\pi_{B}$, and their strategies satisfy the first-order conditions $x_{A}(x)=x+\phi_{A}\left(x_{A}, x_{B}\right)$ and $x_{B}(x)$ $=x-\phi_{B}\left(x_{A}, x_{B}\right)$; here $\phi_{A}, \phi_{B}$ denote the partial derivatives of $\phi$ with respect to $x_{A}$ and $x_{B}$. The arbiter's strategy satisfies $\phi\left(x_{A}, x_{B}\right)=E_{\mu}\left[x \mid x_{A}, x_{B}\right]$. This condition follows from the arbiter adjudicating so as to minimize error costs given his updated beliefs at the information set defined by the observations of $x_{A}$ and $x_{B}$. Here we have again that equilibria are revealing and involve falsification.

Lemma 2. Every equilibrium is revealing and in every equilibrium at least one party falsifies.

To select an equilibrium, we need some more structure. Let the strategies $x_{A}(x), x_{B}(x)$, and $\phi\left(x_{A}, x_{B}\right)$ be part of an equilibrium. First, we restrict attention to reporting strategies $x_{A}(x), x_{B}(x), \mathbb{R} \mapsto \mathbb{R}$, which are strictly increasing. 
Next, consider an out-of-equilibrium pair $\left(x_{A}, x_{B}\right)$, that is, a set of reports such that there does not exist $x$ with $\left(x_{A}, x_{B}\right)=\left(x_{A}(x), x_{B}(x)\right)$. At such an out-ofequilibrium information set, the adjudicator believes that at most one party deviated. A similar restriction on beliefs, termed the "minimality condition," has been used by, for example, Bagwell and Ramey (1991).

The first condition implies that that there exist functions $h\left(x_{A}\right)$ and $g\left(x_{B}\right)$ such that $h\left(x_{A}(x)\right) \equiv g\left(x_{B}(x)\right) \equiv x$. The minimality condition implies that an out-of-equilibrium pair is interpreted in terms of the minimum number of deviations consistent with it. Given these assumptions, the adjudicator's beliefs and his sequentially rational strategy are then

$\phi\left(x_{A}, x_{B}\right)=E\left(x \mid x_{A}, x_{B}\right)=(1-\lambda) h\left(x_{A}\right)+\lambda g\left(x_{B}\right)$.

The beliefs are that with probability $\lambda$ it is $A$ who has deviated and that $B$ did not deviate. Thus, the adjudicator assigns probability $\lambda$ to the true state being $x=g\left(x_{B}\right)$. Similarly, he assigns probability $1-\lambda$ to the true state being $x=$ $h\left(x_{A}\right)$. Note that equation (1) also holds at equilibrium and is consistent with full revelation on the equilibrium path.

Proposition 2. Assume both reports are increasing in $x$ and the minimality condition holds. If both parties testify, the unique minimum falsification cost equilibrium has strategies $x_{A}(x)=x+0.5, x_{B}(x)=x-0.5, \phi\left(x_{A}, x_{B}\right)=0.5 x_{A}+$ $0.5 x_{B}, \mu\left(x_{A}-0.5 \mid x_{A}, x_{B}\right)=1$ if $x_{A}-0.5=x_{B}+0.5$, and otherwise $\mu\left(x \mid x_{A}, x_{B}\right)= \begin{cases}0.5, & \text { for } x=x_{A}-0.5 \\ 0.5, & \text { for } x=x_{B}+0.5\end{cases}$

The expected loss $\bar{L}_{J}=2 \gamma+0.25$.

If both parties are heard, $A$ overstates the true value by 0.5 whereas $B$ understates by this amount. The arbiter's beliefs reflect these incentives and he takes the average of both reports.

Let us now compare the amount of lying under single and joint submissions. The extent of lying by, say, $B$ under single submission is twice the amount of her lying under joint submission. The reason is that under single submission, greater weight is given to the party's report, thereby inducing her to falsify more. Thus, confronting the parties in adversarial hearings induces either of them to distort the evidence less than when only one testimony is heard. Given the quadratic cost of lying, this implies that the total variable cost of distorting is less under joint than under single submissions. Yet when both parties are heard, we have a duplication of the fixed submission cost $\gamma$.

This last observation can be put differently. Along the equilibrium path, the arbiter can infer the truth from the evidence provided by either party. However, when a pair of submissions with $x_{A}-x_{B} \neq 1$ is observed, the arbiter knows that at least one party must have deviated. His beliefs are that only one did so and that a deviation by $A$ is as likely as a deviation by $B$. In turn, this probability of one half determines the extent to which a party's boosting of the evidence can 


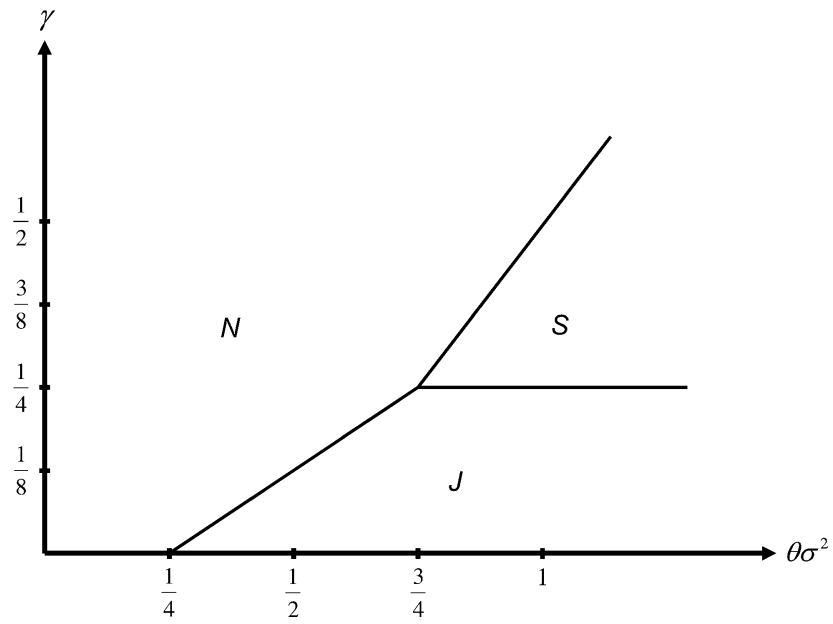

Figure 1. Simultaneous Submissions.

influence the arbiter's beliefs. In effect, under joint submissions, a party has half as much influence as she would if she were alone in submitting evidence.

\subsection{Optimal Number of Parties to Submit Evidence}

Let us now determine the optimal number of parties to submit evidence. The arbiter chooses whether no party $N$, only party $A$ or $B$ under procedure $S$, or both parties $J$ are required to submit evidence so as to minimize the expected $\operatorname{loss} \bar{L}_{d}$.

From the two foregoing results,

$\bar{L}_{J}=2 \gamma+0.25 \leq \gamma+0.5=\bar{L}_{S} \quad$ if $\gamma \leq 0.25$,

that is, joint submissions are cheaper if the fixed submission cost is not too large. Perfect accuracy obtains under either procedure $S$ or $J$. Taking the value of information into account then yields:

Proposition 3.

(i) For $\gamma \leq 0.25$, the optimal procedure is $J$ if $\theta \sigma^{2} \geq 2 \gamma+0.25$ and $N$ otherwise.

(ii) For $\gamma>0.25$, the optimal procedure is $S$ if $\theta \sigma^{2} \geq \gamma+0.5$ and $N$ otherwise.

Figure 1 shows in the $\left(\theta \sigma^{2}, \gamma\right)$ plane the regions where the arbiter requires both, only one, or no party to submit evidence. When the value of information is large (i.e., $\theta \sigma^{2}>0.75$ ), the arbiter requires joint submissions if the fixed submission cost is sufficiently small, single submissions for intermediate values, and hears no one if the fixed cost is large. When $0.25<\theta \sigma^{2}<0.75$, the relevant choice is only between joint submissions or hearing no one: the value of information is 
then not large enough for single submissions to be worth it since they entail too much falsification. Finally, when $\theta \sigma^{2}<0.25$, the value of information is too small even to compensate for the falsification costs under joint submissions.

\section{Alternative Procedures}

We now consider whether alternative institutional arrangements can improve the trade-off between accuracy in adjudication and falsification costs. In a related article, Sobel (1985) also considers an arbiter concerned both with accuracy and litigation expenditures. He points out that the capacity to commit not to adjudicate according to one's beliefs ex post might be useful. ${ }^{8}$

In the first alternative setup we take up this idea, yet we do not consider such a strong form of commitment: our arbiter cannot do otherwise than adjudicate according to his inferences once he is informed of the parties' submissions. We take it, however, that the arbiter may require written reports from the parties while simultaneously committing to some probability of not reading them. When reports are read, the arbiter adjudicates according to the inferences drawn from the parties' submissions. When he remains uninformed, he adjudicates on the basis of his priors, which is also sequentially rational. We show that the arbiter will always commit to a positive probability of remaining uninformed. Moreover, this enlarges the set of parameter values for which submissions are desirable.

In the second alternative setup, we consider a situation where sequential testimonies are feasible; the procedure allows sufficient time for the second testifying party to react to the first party's testimony. With sequential testimonies, there are equilibria where parties do not falsify at all. Accordingly, sequencing enlarges the set of parameter values for which it is preferable to hear both parties, rather than one or none. In either alternative arrangement, we focus on equilibria satisfying the minimality condition discussed in the preceding section.

\subsection{Partial Commitment}

As previously, the parties may be required to present evidence, now in the form of a written report, and they do so simultaneously when both must testify. By contrast with Section 3, however, the arbiter now "announces" a probability $\alpha$ of actually reading the reports; with probability $(1-\alpha)$ he remains uninformed. ${ }^{9}$ Obviously, he adjudicates $\hat{x}=\bar{x}$ when he remains uninformed. Parties required to submit a report, therefore, anticipate the adjudicated value to be

8. In Sobel's analysis, the parties can only submit hard, albeit imperfect evidence (i.e., they cannot falsify), but disclosure is costly and the parties may choose not to disclose the evidence available to them.

9. In Italy, for example, judges are so overloaded with cases that they tend not to read all the presented evidence. In the ongoing criminal Swissair bankruptcy proceedings, one issue is whether the former CEO Mario Corti misinformed the public about the extension of the airline's credit limit at the shareholder meeting on April 21, 2001. The judge Fischer admitted that he did not read the entire (highly complex) contract to extend the airline's credit limit; see Neue Züricher Zeitung 01/24/07, p. 23. 
$\hat{x}=(1-\alpha) \bar{x}+\alpha \phi(\cdot)$,

where, as before, $\phi(\cdot)$ denotes the arbiter's decision in terms of the parties' submissions. For a given $\alpha$, the minimum falsification cost equilibrium satisfying the minimality condition is then a simple variant of the equilibrium described in Proposition 2.

Proposition 4. Assume the arbiter commits to a probability $\alpha$ of reading reports. In a minimum falsification cost equilibrium,

(i) when only $A$ reports, $x_{A}=x+\alpha$ and $\phi\left(x_{A}\right)=x_{A}-\alpha$; when only $B$ does, $x_{B}=x-\alpha$ and $\phi\left(x_{B}\right)=x_{B}+\alpha$; and

(ii) when both parties are required to submit, $x_{A}=x+0.5 \alpha, x_{B}=x-0.5 \alpha$, and $\phi\left(x_{A}, x_{B}\right)=0.5 x_{A}+0.5 x_{B}$.

Compared to Section 3, the parties now falsify less: boosting one's claim has less influence on the arbiter's decision due to the probability that reports will not be read. When they are read, the arbiter infers the truth and adjudicates accordingly. Nevertheless, adjudication is not always accurate because reports are sometimes ignored. A low $\alpha$ leads to little distortion yet to high inaccuracy.

The expected error cost is

$E(l)=\theta(1-\alpha) E(\bar{x}-x)^{2}=\theta(1-\alpha) \sigma^{2}$.

When only one party submits, the social loss as a function of $\alpha$ is

$\bar{L}_{S}(\alpha)=E(l)+C=\theta(1-\alpha) \sigma^{2}+\gamma+0.5 a^{2}$.

Minimizing with respect to $\alpha$ gives the optimal probability under single submission

$a_{S}= \begin{cases}\theta \sigma^{2}, & \text { if } \theta \sigma^{2} \leq 1 \\ 1, & \text { otherwise }\end{cases}$

Under joint submission the social loss is

$\bar{L}_{J}(\alpha)=E(l)+C=\theta(1-\alpha) \sigma^{2}+2 \gamma+0.25 a^{2}$

and the optimal probability is

$a_{J}= \begin{cases}2 \theta \sigma^{2}, & \text { if } 2 \theta \sigma^{2} \leq 1 \\ 1, & \text { otherwise }\end{cases}$

In both cases, the weight given to the parties' reports is increasing in the value of information: the more noisy his priors, the more the arbiter is willing to read the reports. Note that $a_{J}>a_{S}$ whenever $a_{S}<1$. Joint submissions induce fewer distortions than single ones; therefore, a larger $\alpha$ is less costly in terms of falsification costs. It follows that adjudication is, on average, more accurate under joint submissions, except when the value of information is sufficiently large so 


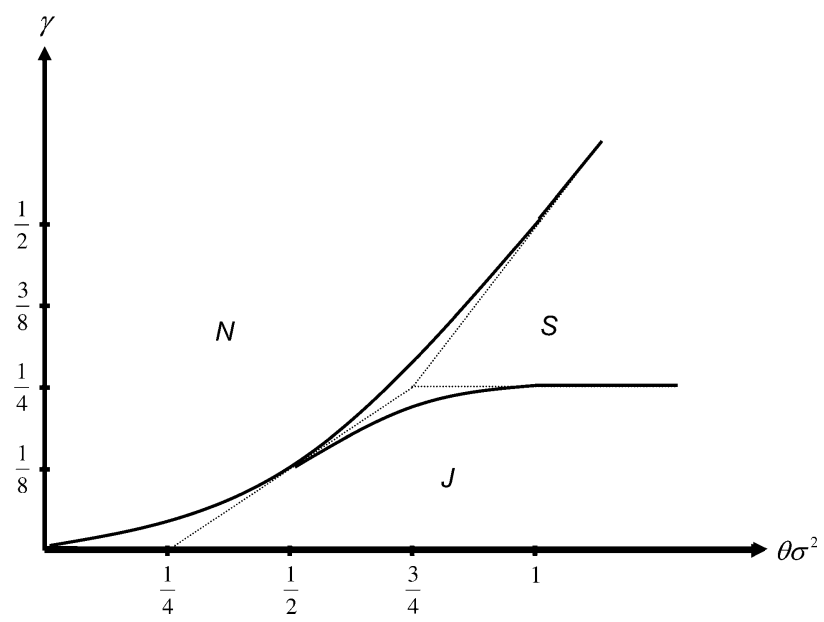

Figure 2. Partial Commitment.

that $a_{S}=1$. The next proposition compares the different procedures given the optimal $\alpha$.

\section{Proposition 5.}

(i) When $\theta \sigma^{2} \leq 0.5$, the optimal procedure is $J$ if $\gamma \leq 0.5 \theta^{2} \sigma^{4}$ and $N$ otherwise.

(ii) When $0.5<\sigma^{2} / \theta \leq 1$, the optimal procedure is $J$ if $\gamma \leq \theta \sigma^{2}-$ $0.5 \theta^{2} \sigma^{4}-0.25, S$ if $\theta \sigma^{2}-0.5 \theta^{2} \sigma^{4}-0.25<\gamma \leq 0.5 \theta^{2} \sigma^{4}$, and $N$ otherwise.

(iii) When $\theta \sigma^{2}>1$, the optimal procedure is as in Proposition 3 .

The corresponding regions are shown in Figure 2. The boundaries for the nocommitment case of Section 3 are reproduced as dotted lines. Compared with the noncommitment case, region $J$ is made larger at the expense of the previous region $N$ and region $S$ is made larger at the expense of the previous regions $J$ and $N$.

By contrast with the previous results, it is now always optimal to require both parties to submit a report when $\gamma$ is sufficiently small. When the fixed costs are negligible, both parties should submit irrespective of the accuracy of the arbiter's prior information. The intuition is straightforward. When $\gamma$ is small, total submission costs are smaller under joint rather than single submissions since each party's testimony is accorded a smaller weight. Moreover, falsification costs depend on the probability $\alpha$ that reports are read and this probability is chosen optimally, given the trade-off between inducing falsification and acquiring information. For larger values of $\gamma$, duplication of costs becomes an issue. The best procedure is then again single submissions, provided information is worth acquiring at all. 


\subsection{Sequential Hearings}

We now revert to the noncommitment setup, but allow the parties to present their submissions sequentially. The simultaneous-move game of Section 3 can be justified if, say, testimony preparations take so much time that even if one party testifies before the other the latter may not react. We henceforth relax this assumption. The arbiter chooses who is to testify, and if both parties are called upon, who should go first. The follower observes the first testimony and adjusts her own accordingly. Without loss of generality, let $A$ be the party testifying first. The parties' strategies are then $x_{A}(x)$ and $x_{B}\left(x, x_{A}\right)$. As before, the arbiter's strategy is $\phi\left(x_{A}, x_{B}\right)$.

The parties' strategies satisfy

$$
\begin{aligned}
& x_{B}\left(x, x_{A}\right) \in \underset{x_{B}}{\arg \max } \pi_{B}=-\phi\left(x_{A}, x_{B}\right)-0.5\left(x_{B}-x\right)^{2}, \\
& x_{A}(x) \in \underset{x_{A}}{\arg \max } \pi_{A}=\phi\left(x_{A}, x_{B}\left(x, x_{A}\right)\right)-0.5\left(x_{A}-x\right)^{2} .
\end{aligned}
$$

The first-order condition for $B$ 's optimization problem is the same as with simultaneous hearings, that is,

$\phi_{B}\left(x_{A}, x_{B}\right)=x-x_{B}$,

but $A$ 's is now

$\phi_{A}\left(x_{A}, x_{B}\left(x, x_{A}\right)\right)+\phi_{B}\left(x_{A}, x_{B}\left(x, x_{A}\right)\right) \partial x_{B}\left(x, x_{A}\right) / \partial x_{A}=x_{A}-x$.

Again there are multiple equilibria. In particular, the strategies described in Proposition 2 remain an equilibrium, in which case obviously $\partial x_{B} / \partial x_{A}=0$. However, new possibilities are introduced by the fact that $A$ is now in the position of a Stackelberg leader. If, say, exaggerated claims by $A$ provoke $B$ into boosting her own claims, that is, if $\partial x_{B} / \partial x_{A}<0$, it may be that $A$ will falsify less compared to simultaneous submissions. The intuition is that provocative claims by $A$ may be a waste of money if they induce similarly exaggerated claims by $B$, with little net effect on the arbiter's decision. Looking as before for a minimum falsification cost equilibrium, we find that sequencing can actually eliminate falsification completely.

Proposition 6. With sequential hearings, there exists an equilibrium such that along the equilibrium path $x_{A}=x_{B}=x$.

In this equilibrium party $A$ always reports the truth, that is, $x_{A}=x . B$ also reports the truth, given $A$ did not overreport. If, however, $A$ overreports, $B$ retaliates by underreporting, that is, $x_{B}<x$. See the reaction functions in Figure 3.

The arbiter's beliefs and adjudication function are as follows: Along the equilibrium path when both parties report the same value, he believes that both spoke the truth and adjudicates the correct value. When $x_{A}>x_{B}$, he believes that $A$ deviated and $B$ did not. Accordingly, he adjudicates the $x$ giving rise to 


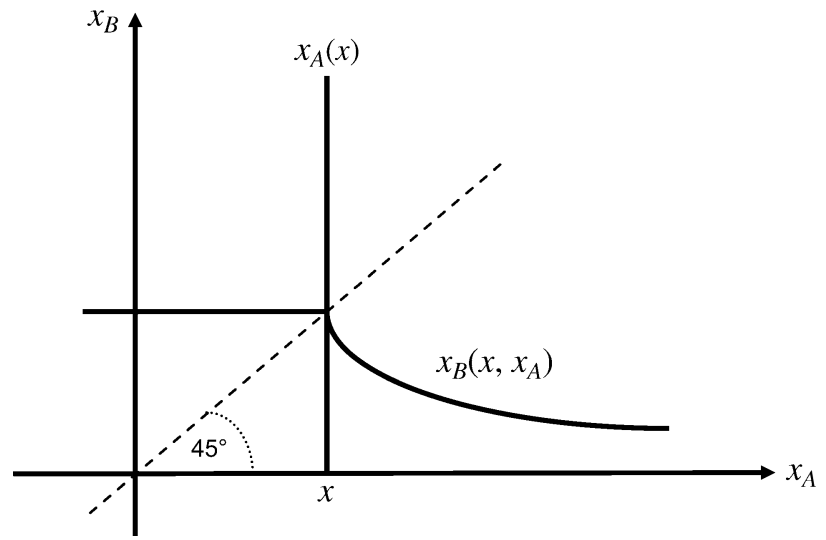

Figure 3. Reaction Functions Under Sequential Submissions.

$B$ 's message $x_{B}$. Conversely, when $x_{A}<x_{B}$, he believes that $B$ deviated and $A$ did not. He, therefore, adjudicates the $x$ leading to $A$ 's report $x_{A}$. As we show in the Appendix, the parties' reporting strategies are indeed best responses to the arbiter's adjudication strategy.

It is, therefore, possible to construct an equilibrium under sequential hearings where both parties report truthfully. If $B$ boosts her own claims in the specified way if provoked by exaggerated reports by $A, A$ has no incentive to overreport. ${ }^{10}$ Recall, however, that the strategies of our simultaneous hearing equilibrium also form an equilibrium under sequential hearings, with the same amount of distortions as in Proposition 2. It is, therefore, unclear why the parties should coordinate on this particular truth-telling equilibrium, although one can make an argument that it minimizes their submission costs. The equilibrium has the equilibrium path described in Proposition 6 if we again select the least-cost signaling equilibrium given monotonic strategies and the minimality condition.

We can now examine again whether both, only one, or no party should testify. Obviously, the effect of sequencing is to reduce the cost associated with hearing both parties. Thus, compared to simultaneous hearings, the parameter region for procedure $J$ is enlarged at the expense of $S$ and $N$.

\section{Proposition 7.}

(i) When $\theta \sigma^{2} \leq 1$, the optimal procedure is $J$ if $\gamma \leq 0.5 \theta \sigma^{2}$ and $N$ otherwise.

(ii) When $\theta \sigma^{2}>1$, the optimal procedure is $J$ if $\gamma \leq 0.5, S$ if $0.5<\gamma \leq$ $\theta \sigma^{2}-0.5$, and $N$ otherwise.

The regions for each procedure are presented in Figure 4. The dotted lines depict the previous regions under simultaneous hearings without commitment.

10. The result is reminiscent of the usefulness of stage mechanisms in the theory of implementation when agents have correlated information. See, for instance, Moore and Repullo (1987) and Ma (1988). 


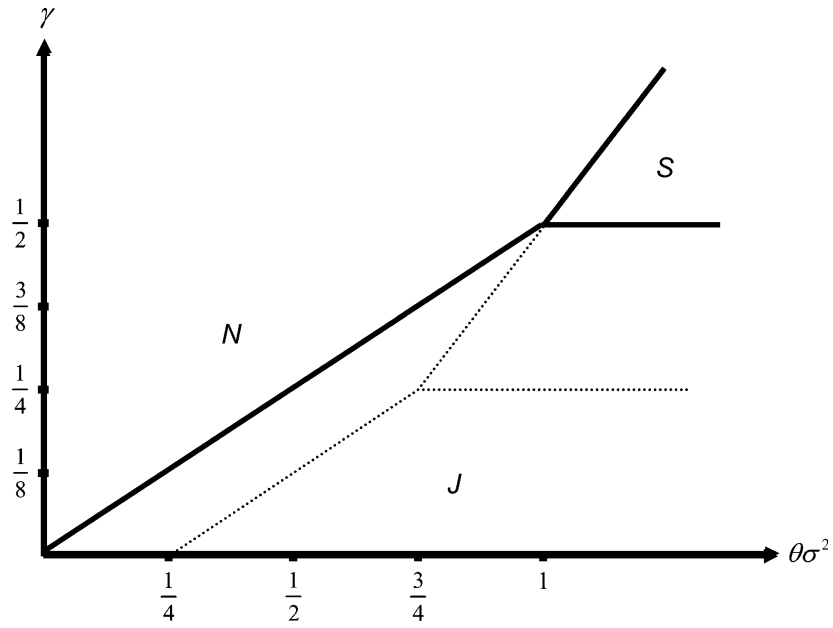

Figure 4. Sequential Submissions.

Although falsification costs are not an issue with sequential testimonies (given the appropriate equilibrium), the overall conclusion is qualitatively the same as in the previous setups. Hearing both parties induces less falsification than hearing only one. Thus, both parties should be heard unless the fixed cost of testifying is sufficiently large.

\section{Concluding Remarks}

In this article, we analyze a stylized model of the trade-off between accuracy in adjudication and misrepresentation costs. We show that the cost of misrepresentation (net of fixed submission costs) is lower when both parties are heard than when only one party submits evidence: hearing both parties duplicates fixed submission costs but lowers misrepresentation costs. Accordingly, it is preferable to hear both parties when fixed costs are low. We, therefore, qualify Tullock's (1975) statement that adversarial systems are inferior to inquisitorial systems due to the duplication of misrepresentation costs.

We also point out the usefulness of commitment. When the judge can commit not to infer and adjudicate the truth from the parties' statements, it is more likely that he hears testimony. We also show that the sequencing of testimonies may be useful when it is feasible to allow parties to react to one another. Sequential testimony may eliminate falsification altogether and, therefore, also makes joint submissions more attractive.

A few qualifications and remarks are in order. The quadratic cost functions allowed us to obtain closed form solutions. Our conjecture is that most of our results also hold under more general falsification cost functions. The parties were assumed to be perfectly informed. However, it could be that they observe the true state with error and can falsify with respect to what they observed. If the parties' observation errors are not perfectly correlated, hearing two rather than 
one party would then yield more information. When fixed submission costs are not too large, this would therefore provide an additional reason for choosing joint rather than single submissions. Another extension would be to consider the case where the arbiter is unsure about the parties' capacity to falsify. We conjecture that this would also make joint submissions more advantageous.

Our results are driven by the fact that the arbiter can only adjudicate one value that one party loses and the other party gains. If we relax this adding-up constraint, the arbiter could obviously do better. The judge could use, for example, the following mechanism: if both parties make the same report, he adjudicates this value. If the parties report different values, the judge punishes both of them heavily for perjury. ${ }^{11}$ In reality, however, perjury cases are very rare and there is plenty of evidence indicating that slanted testimony is endemic in courts. ${ }^{12}$ Since perjury law seems to be ineffective, we did not include this possibility in the adjudication function. Moreover, nonjudicial proceedingsfor example, regulatory hearings - usually have no such provisions.

\section{Appendix}

Proof of Lemma 1. If the equilibrium with strategies $x_{A}(x)$ and $\phi\left(x_{A}\right)$ is not revealing, there must exist $x^{\prime} \neq x^{\prime \prime}$ such that $x_{A}\left(x^{\prime}\right)=x_{A}\left(x^{\prime \prime}\right)=x_{A}$. Yet the equilibrium strategy must satisfy $\phi^{\prime}\left(x_{A}\right)=x_{A}-x^{\prime}=x_{A}-x^{\prime \prime}$ which cannot hold for $x^{\prime} \neq x^{\prime \prime}$.

To show falsification suppose on the contrary that $A$ never falsifies, that is, $x_{A}(x)=x$ for all $x$. Then $\phi\left(x^{\prime}\right)=x^{\prime}$ for all $x^{\prime}$, that is, the adjudicator must infer that $x=x^{\prime}$ when the party submits $x^{\prime}$. However, given the adjudicator's response, $A$ 's equilibrium strategy would then be $x_{A}=x+1$, a contradiction.

Q.E.D.

Proof of Proposition 1. If the judge hears only party $A$ and adjudicates $\hat{x}=\phi\left(x_{A}\right), A$ maximizes $\pi_{A}=\phi\left(x_{A}\right)-0.5\left(x_{A}-x\right)^{2}$. The first-order condition gives us $x_{A}=x+\phi^{\prime}\left(x_{A}\right)$. To have a global maximum the second-order condition $\phi^{\prime \prime}\left(x_{A}\right)<1$ must be satisfied for all $x_{A} \in \mathbb{R}$. Truth revelation requires $x=\phi\left(x_{A}\right)$. Plugging this into the first-order condition gives us the differential equation

$x_{A}=\phi\left(x_{A}\right)+\phi^{\prime}\left(x_{A}\right)$,

which has the general solution (see e.g., Chiang 1984, 481)

$\phi\left(x_{A}\right)=\mathrm{e}^{-\left(x_{A}+k_{1}\right)}\left[k_{2}+\int x_{A} \mathrm{e}^{x_{A}+k_{1}} \mathrm{~d} x_{A}\right]$,

with $k_{1}$ and $k_{2}$ constant; solving yields with $k_{3}$ as another constant

11. See Demski and Sappington (1984) for an analysis of information extraction in a multiagent context.

12. For example, in a continuing scandal in New York City, police engaged in a pattern of perjury so common that they called it "testilying"; in impeachment proceedings former President Clinton admitted making misleading statements about his sexual conduct while steadfastly denying that he committed perjury. For more evidence on slanted testimony, see Cooter and Emons (2003). 


$$
\left(\mathrm{e}^{-k_{2}} k_{1}+k_{3}\right) \mathrm{e}^{-x_{A}}+x_{A}-1:=K \mathrm{e}^{-x_{A}}+x_{A}-1,
$$

where $K$ is a constant. The second-order condition can only be satisfied for all $x_{A}$ for $K \leq 0$. Hence, the unique solution to our problem is

$\phi\left(x_{A}\right)=K \mathrm{e}^{-x_{A}}+x_{A}-1$

with $K \leq 0$. Any beliefs of the judge given by this equation have the desired properties. To further pin down beliefs, we pick the equilibrium which minimizes submission costs. This means we minimize $\left(x_{A}-x\right)^{2}$ and this is achieved by $K=0$. A similar reasoning gives us $\phi\left(x_{B}\right)=K \mathrm{e}^{x_{B}}+x_{B}+1$ with $K \geq 0$; again we set $K=0$.

Q.E.D.

The proof of Lemma 2 is along the same lines as the proof of Lemma 1 and is, therefore, omitted.

Proof of Proposition 2. On the equilibrium path, using (1),

$x_{A}(x)-x=\phi_{A}\left(x_{A}(x), x_{B}(x)\right)=(1-\lambda) h^{\prime}\left(x_{A}(x)\right)$,

$x-x_{B}(x)=\phi_{B}\left(x_{A}(x), x_{B}(x)\right)=\lambda g^{\prime}\left(x_{B}\right)$,

$x=\phi\left(x_{A}(x), x_{B}(x)\right)=h\left(x_{A}(x)\right)=g\left(x_{B}(x)\right)$.

Thus, in equilibrium,

$x_{A}-h\left(x_{A}\right)=(1-\lambda) h^{\prime}\left(x_{A}\right)$,

$g\left(x_{B}\right)-x_{B}=\lambda g^{\prime}\left(x_{B}\right)$.

The general solutions to these differential equations are for some constants $k_{A}$ and $k_{B}$,

$h\left(x_{A}\right)=k_{A} \mathrm{e}^{-x_{A} /(1-\lambda)}+x_{A}-(1-\lambda)$,

$g\left(x_{B}\right)=k_{B} \mathrm{e}^{x_{B} / \lambda}+x_{B}+\lambda$.

The second-order necessary conditions of the parties' optimization problems are $\phi_{A A} \leq 1$ and $\phi_{B B} \geq-1$. We therefore require

$\phi_{A A}\left(x_{A}, x_{B}\right)=(1-\lambda) h^{\prime \prime}\left(x_{A}\right)=k_{A} \mathrm{e}^{-x_{A} /(1-\lambda)} /(1-\lambda) \leq 1$,

$\phi_{B B}\left(x_{A}, x_{B}\right)=\lambda g^{\prime}\left(x_{B}\right)=k_{B} \mathrm{e}^{x_{B} / \lambda} / \lambda \geq-1$.

This implies $k_{A} \leq 0$ and $k_{B} \geq 0$ if the conditions are to hold for all $x_{A}$ and $x_{B}$.

Finally, falsification costs on the equilibrium path are equal to

$$
\begin{aligned}
0.5\left(x_{A}-h\left(x_{A}\right)\right)^{2}+0.5\left(g\left(x_{B}\right)-x_{B}\right)^{2}= & 0.5\left(1-\lambda-k_{A} \mathrm{e}^{-x_{A} /(1-\lambda)}\right)^{2} \\
& +0.5\left(\lambda+k_{B} \mathrm{e}^{x_{B} / \lambda}\right)^{2} .
\end{aligned}
$$


Given $k_{A} \leq 0$ and $k_{B} \geq 0$, falsification costs are minimal at all equilibrium pairs if $k_{A}=k_{B}=0$ and $\lambda=1 / 2$. The minimum falsification cost equilibrium consistent with assumptions 1 and 2 is therefore characterized by

$x_{A}(x)-x=x-x_{B}(x)=0.5$

and

$\phi\left(x_{A}, x_{B}\right)=0.5\left(x_{A}-0.5\right)+0.5\left(x_{B}+0.5\right)=0.5 x_{A}+0.5 x_{B}$.

Q.E.D.

Proposition 3 as well as Propositions 5 and 7 follow directly from comparing the social losses under no, single, and joint submissions.

Proof of Proposition 4. The proof for single submissions is left to the reader. For joint submissions, the argument is similar to that of Proposition 2. Any equilibrium pair $\left(x_{A}, x_{B}\right)$ satisfies

$x_{A}=\phi\left(x_{A}, x_{B}\right)+a \phi_{A}\left(x_{A}, x_{B}\right)$ and $x_{B}=\phi\left(x_{A}, x_{B}\right)-a \phi_{B}\left(x_{A}, x_{B}\right)$.

For a given $x_{B}$, the solutions to the first differential equation are

$\phi=K\left(x_{B}\right) \mathrm{e}^{-x_{A} / a}+x_{A}-a$.

Plugging into the second differential equation gives

$x_{B}=\phi\left(x_{A}, x_{B}\right)-\phi_{B}\left(x_{A}, x_{B}\right)=K\left(x_{B}\right) \mathrm{e}^{-x_{A} / a}+x_{A}-a-\left(K^{\prime}\left(x_{B}\right) \mathrm{e}^{-x_{A}}\right)$,

which yields

$K^{\prime}\left(x_{B}\right)-K\left(x_{B}\right)=\left(x_{A}-x_{B}-a\right) \mathrm{e}^{x_{A} / a}$.

This holds for different $x_{A}$ 's only if $x_{A}-x_{B}=a$ and $K^{\prime}\left(x_{B}\right)-K\left(x_{B}\right)=0$. Hence, $K\left(x_{B}\right)=k \mathrm{e}^{x_{B} / a}$ with $k$ a constant. Consequently,

$\phi=k \mathrm{e}^{\left(x_{B}-x_{A}\right) / a}+x_{A}-a$.

Substituting in (A1) and using $\phi\left(x_{A}, x_{B}\right)=x$ yields

$x_{A}-x=a-k \mathrm{e}^{\left(x_{B}-x_{A}\right) / a} \quad$ and $\quad x-x_{B}=k \mathrm{e}^{\left(x_{B}-x_{A}\right) / a}$

with $x_{A}-x_{B}=a$. Thus, every equilibrium pair satisfies

$x_{A}-x=a-d$ and $x-x_{B}=d$,

where $d:=k \mathrm{e}^{-1}$. Falsification costs at all equilibrium pairs are therefore

$0.5\left(x_{A}-x\right)^{2}+0.5\left(x-x_{B}\right)^{2}=0.5(1-d)^{2}+0.5 d^{2}$.

and are minimized by $d=0.5 a$, yielding $x_{A}=x+0.5 a$ and $x_{B}=x-0.5 a$.

Beliefs when $x_{A}-x_{B}=a$ are 
$\mu\left(\left\{x=x_{A}-0.5 a\right\} \mid x_{A}, x_{B}\right)=\mu\left(\left\{x=x_{B}+0.5 a\right\} \mid x_{A}, x_{B}\right)=1$,

leading to $\phi\left(x_{A}, x_{B}\right)=x_{A}-0.5 a=x_{B}+0.5 a$.

Q.E.D.

Proof of Proposition 6. As a preliminary step, consider first the following equation in the variables $u$ and $v$,

$u=1-e^{-(v+u)}, \quad v \geq 0$.

The solution is a function $u=s(v)$ satisfying $s(0)=0, s(\infty)=1, s^{\prime}(v)>0$, and $s^{\prime}(0)=\infty$. We will use this function throughout in the proof.

In the candidate equilibrium, the parties' strategies are

$x_{A}(x)=x$,

$x_{B}\left(x, x_{A}\right)= \begin{cases}x-s\left(x_{A}-x\right), & \text { if } x_{A} \geq x \\ x, & \text { otherwise }\end{cases}$

These strategies yield $x_{A}=x_{B}=x$ along the equilibrium path.

To characterize the adjudicator's strategy, define

$g\left(x_{A}, x_{B}\right):=x_{B}+1-\mathrm{e}^{-\left(x_{A}-x_{B}\right)}$.

Note that $g(k, k)=k$. Moreover, (A3) can be rewritten as

$g\left(x_{A}, x_{B}\right)=x_{B}+1-\mathrm{e}^{-\left[\left(x_{A}-x\right)+\left(x-x_{B}\right)\right]}$.

From (A2), if $x_{A} \geq x$,

$g\left(x_{A}, x_{B}\left(x, x_{A}\right)\right)=x-s\left(x_{A}-x\right)+1-\mathrm{e}^{-\left[\left(x_{A}-x\right)+s\left(x_{A}-x\right)\right]}$.

From the definition of $s(\cdot)$, it follows that

$g\left(x_{A}, x_{B}\left(x, x_{A}\right)\right)=x, \quad$ all $x_{A} \geq x$.

In the candidate equilibrium, the adjudicator's strategy is

$\phi\left(x_{A}, x_{B}\right)= \begin{cases}g\left(x_{A}, x_{B}\right), & \text { if } x_{A} \geq x_{B} \\ x_{A}, & \text { otherwise }\end{cases}$

We now characterize the beliefs supporting this strategy. When observing $x_{A}=x_{B}$, the adjudicator's beliefs are that $x=x_{A}=x_{B}$. These beliefs are correct along the equilibrium path. The adjudicator's decision $\phi\left(x_{A}, x_{B}\right)$ is then sequentially rational. The observation of $x_{A} \neq x_{B}$ is out of equilibrium. The adjudicator then believes that one party, and only one, deviated from his equilibrium strategy. When $x_{B}>x_{A}$, he believes that for sure the deviating party is $B$; hence he adjudicates $x_{A}$. When $x_{B}<x_{A}$, he believes that for sure the deviating party is $A$. Given (A4), the adjudicator must then infer that the true $x$ is $g\left(x_{A}, x_{B}\right)$, hence it is again sequentially rational to adjudicate as specified in (A5). 
It remains to show that $x_{A}(x)$ and $x_{B}\left(x, x_{A}\right)$ are indeed the parties' equilibrium strategies, that is, that

$x_{A}(x) \in \arg \max \pi_{A}:=\phi\left(x_{A}, x_{B}\left(x, x_{A}\right)\right)-0.5\left(x_{A}-x\right)^{2}$,

$x_{B}\left(x, x_{A}\right) \in \underset{x_{B}}{\arg \max } \pi_{B}:=-\phi\left(x_{A}, x_{B}\right)-0.5\left(x_{B}-x\right)^{2}$.

We start with party $B$. Differentiating $B$ 's payoff function gives us

$\frac{\partial \pi_{B}}{\partial x_{B}}= \begin{cases}-\left(1-\mathrm{e}^{-\left(x_{A}-x_{B}\right)}\right)-x_{B}+x, & \text { if } x_{A} \geq x_{B} \\ -x_{B}+x, & \text { otherwise. }\end{cases}$

The derivative is continuous in $x_{B}$. Moreover,

$\frac{\partial^{2} \pi_{B}}{\partial x_{B}^{2}}= \begin{cases}\mathrm{e}^{-\left(x_{A}-x_{B}\right)}-1, & \text { if } x_{A} \geq x_{B} \\ -1, & \text { otherwise }\end{cases}$

Observe that $\partial^{2} \pi_{B} / \partial x_{B}^{2} \leq 0$ with strict inequality except at $x_{A}=x_{B}$. Hence, $\pi_{B}$ is strictly concave in $x_{B}$, implying a maximum at the unique value of $x_{B}$ where the first-order derivative vanishes. To determine this value, write $u=x-x_{B}$ and $v=x_{A}-x$, so that

$\frac{\partial \pi_{B}}{\partial x_{B}}= \begin{cases}-\left(1-\mathrm{e}^{-(v+u)}\right)+u, & \text { if } u+v \geq 0 \\ u, & \text { otherwise }\end{cases}$

When $v \geq 0$, the derivative vanishes at $u=s(v) \geq 0$. When $v<0$, it vanishes at $u=0$. This proves that $x_{B}\left(x, x_{A}\right)$ as defined in (A2) is $B$ 's best reply.

Consider now party $A$ 's strategy. If $x_{A}<x$, party $B$ will play $x_{B}=x$ so that $\phi\left(x_{A}, x_{B}\right)=x_{A}$. Hence $A$ 's payoff is then

$\pi_{A}=x_{A}-0.5\left(x_{A}-x\right)^{2}$,

which is strictly increasing in $x_{A}$ for $x_{A}<x$. If $x_{A} \geq x$, party $B$ will play $x_{B}=x-$ $s\left(x_{A}-x\right) \leq x_{A}$. Party $A$ 's payoff is then

$\pi_{A}=g\left[x_{A}, x_{B}\left(x, x_{A}\right)\right]-0.5\left(x_{A}-x\right)^{2}=x-0.5\left(x_{A}-x\right)^{2}$,

which is strictly decreasing in $x_{A}$ for $x_{A}>x$. Thus, party $A$ 's best play is $x_{A}=x$, proving that $x_{A}(x)$ as defined above is $A$ 's equilibrium strategy. Q.E.D.

\section{References}

Bagwell, Kyle, and Garey Ramey. 1991. "Oligopoly Limit Pricing," 22 Rand Journal of Economics $155-72$.

Bernardo, Antonio E., Eric Talley, and Ivo Welch. 2000. “A Theory of Legal Presumptions,” 16 Journal of Law, Economics, \& Organization 1-49.

Chiang, Alpha C. 1984. Fundamental Methods of Mathematical Economics. Auckland, New Zealand: McGraw-Hill. 
Cooter, Robert, and Winand Emons. 2003. "Truth-Revealing Mechanisms for Courts,” 159 Journal of Institutional and Theoretical Economics 259-79.

Cooter, Robert, and Daniel L. Rubinfeld. 1989. "Economic Analysis of Legal Disputes and Their Resolution," 27 Journal of Economic Literature 1067-97.

Crocker, Keith J., and John Morgan. 1998. "Is Honesty the Best Policy? Curtailing Insurance Fraud Through Optimal Incentive Contracts," 106 Journal of Political Economy 355-75.

Daughety Andrew F., and Jennifer F. Reinganum. 2000a. "On the Economics of Trials: Adversarial Process, Evidence, and Equilibrium Bias,” 16 Journal of Law, Economics, \& Organization 365-94.

- 2000b. “Appealing Judgments,” 31 Rand Journal of Economics 502-26.

Demski, Joel S., and David E. M. Sappington. 1984. “Optimal Incentive Contracts with Multiple Agents," 33 Journal of Economic Theory 152-71.

Dewatripont, Mathias, and Jean Tirole. 1999. “Advocates,” 107 Journal of Political Economy $1-39$.

Farmer, Amy, and Paul Pecorino. 1999. "Legal Expenditure as a Rent-Seeking Game," 100 Public Choice 271-88.

Fluet, Claude, and Paolo Garella. 2002. “Advertising and Prices as Signals of Quality in a Regime of Price Rivalry,” 20 International Journal of Industrial Organization 907-30.

Froeb, Luke, and Bruce Kobayashi. 1996. "Naive, Biased, yet Bayesian: Can Juries Interpret Selectively Produced Evidence?," 12 Journal of Law, Economics, \& Organization 257-76.

. 2001. "Evidence Production in Adversarial vs. Inquisitorial Regimes," 72 Economics Letters 267-72.

Hetzendorf Mark N., and Per B. Overgaard. 2001. "Price Competition and Advertising Signals: Signaling by Competing Senders," 10 Journal of Economics and Management Strategy 621-62.

Katz, Avery. 1988. “Judicial Decisionmaking and Litigation Expenditure,” 8 International Review of Law and Economics 127-43.

Kim, Jeong-Yoo. 2003. "Signal Jamming in Games with Multiple Senders," 3 B.E. Journal of Theoretical Economics, Contributions 7.

Lacker, Jeffrey M., and John A. Weinberg. 1989. "Optimal Contracts Under Costly State Falsification," 97 Journal of Political Economy 1345-63.

Lewis, Tracy, and Michael Poitevin. 1997. "Disclosure of Information in Regulatory Proceedings," 13 Journal of Law, Economics, \& Organization 50-73.

Ma, Ching-To A. 1988. "Unique Implementation of Incentive Contracts with Many Agents," 50 Review of Economic Studies 555-72.

Maggi, Giovanni, and Andrés Rodríguez-Clare. 1995 “Costly Distortion of Information in Agency Problems,” 26 Rand Journal of Economics 675-89.

Milgrom, Paul. 1988. "Employment Contracts, Influence Activities, and Efficient Organization Design," 96 Journal of Political Economy 42-60.

Milgrom, Paul, and John Roberts. 1986. "Relying on the Information of Interested Parties," 17 Rand Journal of Economics 18-32.

Moore, John, and Rafael Repullo. 1987. "Implementation by Stage Mechanisms," 31 European Economic Review 336-41.

Palumbo, Giuliana. 2001. "Trial Procedures and Optimal Limits on Proof-Taking," 21 International Review of Law and Economics 309-27.

Parisi, Francesco. 2002. "Rent-Seeking Through Litigation: Adversarial and Inquisitorial Systems Compared," 22 International Review of Law and Economics 193-216.

Posner, Richard A. 1973. "An Economic Approach to Legal Procedure and Judicial Administration," 2 Journal of Legal Studies 399-458.

- 1999. "An Economic Approach to the Law of Evidence," 51 Stanford Law Review $1477-546$

Riley, John. 2001. "Silver Signals: Twenty-Five Years of Screening and Signaling," 39 Journal of Economic Literature 432-78.

Rubinfeld, Daniel L., and David E. M. Sappington. 1987. "Efficient Awards and Standards of Proof in Judicial Proceedings," 18 Rand Journal of Economics 308-15. 
Sanchirico, Chris W. 2001. "Relying on the Information of Interested - and Potentially Dishonest-Parties," 3 American Law and Economics Review 320-57.

Shin, Hyun S. 1998. "Adversarial and Inquisitorial Procedures in Arbitration," 29 Rand Journal of Economics 378-405.

Sobel, Joel. 1985. "Disclosure of Evidence and Resolution of Disputes: Who Should Bear the Burden of Proof?," in A.E. Roth, ed., Game Theoretic Models of Bargaining. Cambridge: Cambridge University Press, 341-61.

Tullock, Gordon. 1975. "On the Efficient Organization of Trials," 28 Kyklos 745-62.

- 1980. Trials on Trials: The Pure Theory of Legal Procedure. New York, N.Y.: Columbia University Press. 